\title{
¿Sabías que el japonés tiene miles de onomatopeyas?
}

\author{
Elisa Akemi Shimazaki Miho
}

\section{Resumen}

Las onomatopeyas son esenciales en el uso del idioma japonés; sin embargo, es un tema poco incluido durante el proceso de aprendizaje. Este trabajo es una propuesta para apoyar a los estudiantes de idioma japonés de la Escuela Nacional de Lenguas, Lingüística y Traducción (enAlLT) de la Universidad Nacional Autónoma de México (UNAM). Próximamente se incluirá este tipo de material digital en las redes sociales de la mediateca de la enAlLt.

Palabras clave: onomatopeya, idioma japonés, aprendizaje de idiomas.

\section{DiD YOU KNOW THAT JAPANESE HAS THOUSANDS OF ONOMATOPOEIAS?}

\begin{abstract}
Onomatopoeias are essential in the usage of Japanese language; however, it is a topic little explored during the learning process. This is a proposal to support the students of Japanese language of the ENALlT at the National Autonomous University of Mexico (UNAM). This type of digital material will soon be included on the social networks of EnALLT's media library.
\end{abstract}

Keywords: onomatopoeia, Japanese language, language learning.

Recepción: 04/02/2021. Aprobación: 18/03/2021. Dol: http://doi.org/10.22201/cuaieed.16076079e.2021.22.3.13 
"¿Sabías que el japonés tiene miles de onomatopeyas?"

Elisa Akemi Shimazaki Miho

Vol. 22, Núm. 3, mayo-junio 2021

Revista Digital Universitaria

\section{Elisa Akemi Shimazaki Miho}

akemi.shimazaki@enallt.unam.mx

Es profesora de asignatura "A" y asesora de la mediateca de la Escuela Nacional de Lenguas, Lingüística y Traducción (ENALLT) de la Universidad Nacional Autónoma de México (UNAM). Comenzó con la enseñanza del japonés desde 2007. Desde 2015 es profesora de asignatura en la enALLT, UNAM. Directora de la Escuela de Idioma Japonés de la Asociación México Japonesa, A. C., desde 2016. Realizó estudios sobre enseñanza del japonés como lengua heredada en la Japan International Cooperation Agency, Yokohama, y en la Universidad de Kanazawa, Japón. Ha participado como ponente en México, Centroamérica y Sudamérica. Está involucrada en la difusión de la cultura japonesa entre los aprendices de japonés y español. 
Imagen 1. Korokoro. Onomatopeya al rodar un objeto.
Incluso desde antes del inicio de la pandemia por la covid-19, en la mediateca de la Escuela Nacional de Lenguas, Lingüística y Traducción (ENALLT) de la Universidad Nacional Autónoma de México (UNAM), se ha estado realizando un gran esfuerzo para crear materiales digitales de apoyo, que faciliten el estudio autónomo de los estudiantes de idiomas, y el caso del japonés no es la excepción.

En este proyecto se propone crear material sobre las onomatopeyas, pues es un tema poco abarcado dentro de la enseñanza, que, sin embargo, es esencial en el aprendizaje del japonés.
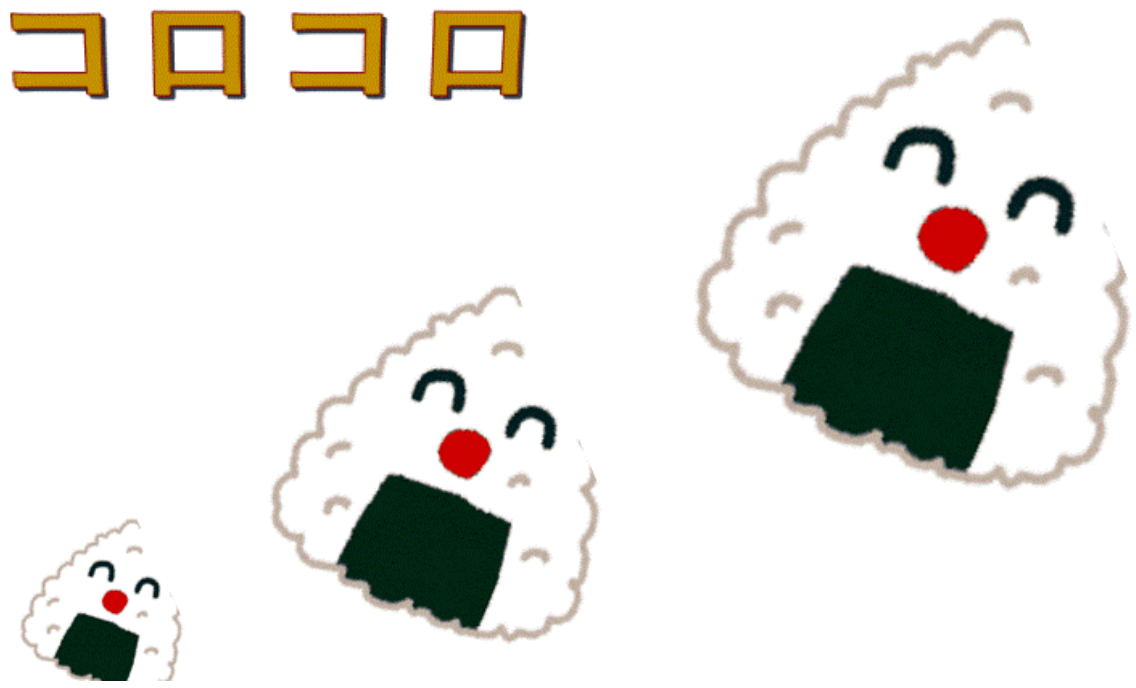

El japonés es una lengua que utiliza mucho simbolismo, no solamente en la escritura a través de los kanji —ideogramas de origen chino, que son similares a los jeroglíficos egipcios o mayas-, sino también mediante los sonidos al hablar.
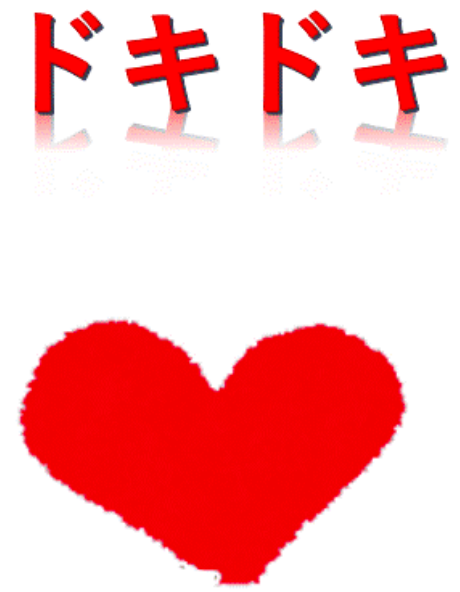

Imagen 2. Doki doki. Sonido de palpitación del corazón.

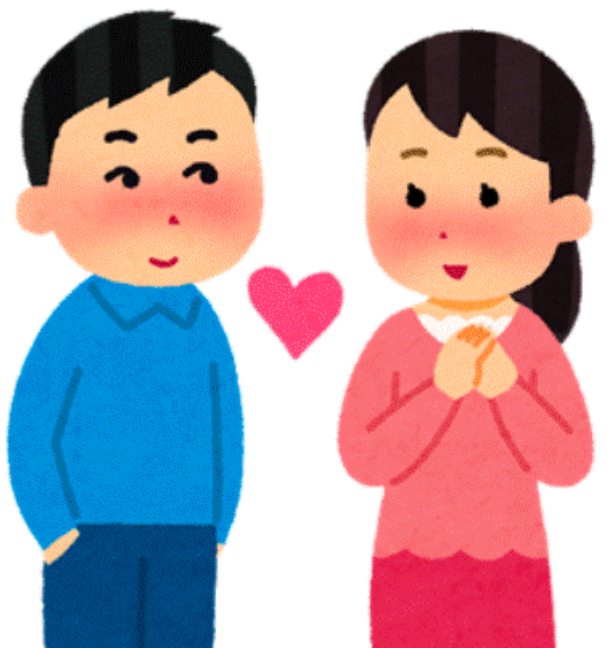


Imagen 3. Guru guru. Onomatopeya usada para decir que algo está rodando o dando vueltas.

Imagen 4. Pyon pyon. Sonido de cuando se salta.
Según la definición del Diccionario de la lengua española, una onomatopeya es una "palabra cuya forma fónica imita el sonido de aquello que designa" (Real Academia Española, 2014).

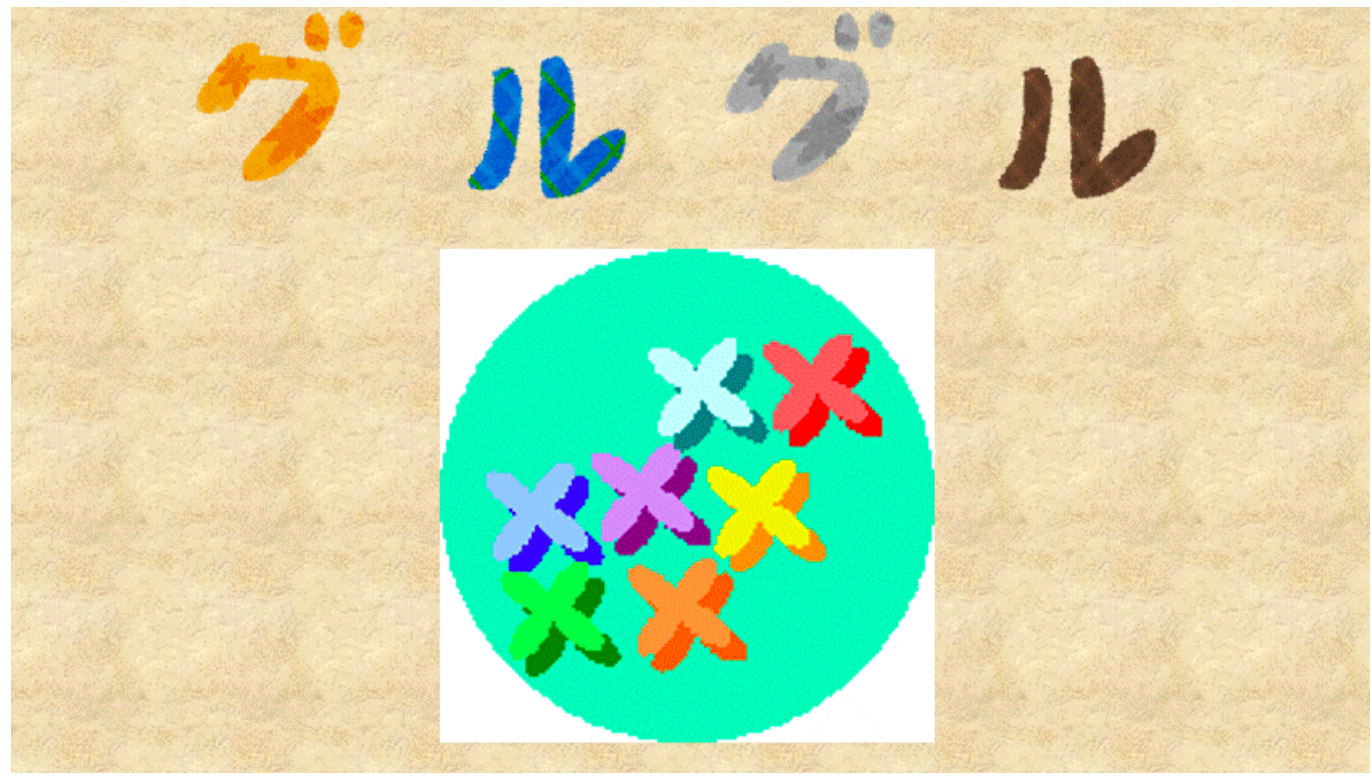

Las onomatopeyas existen en todas las lenguas; sin embargo, en el japonés existen miles y adquieren otra dimensión pues no sólo imitan los sonidos de animales y de personas, o de la naturaleza y de objetos, sino también los sonidos que hacen al moverse. Además, también representan sentimientos y emociones; condiciones o estados de ánimo.

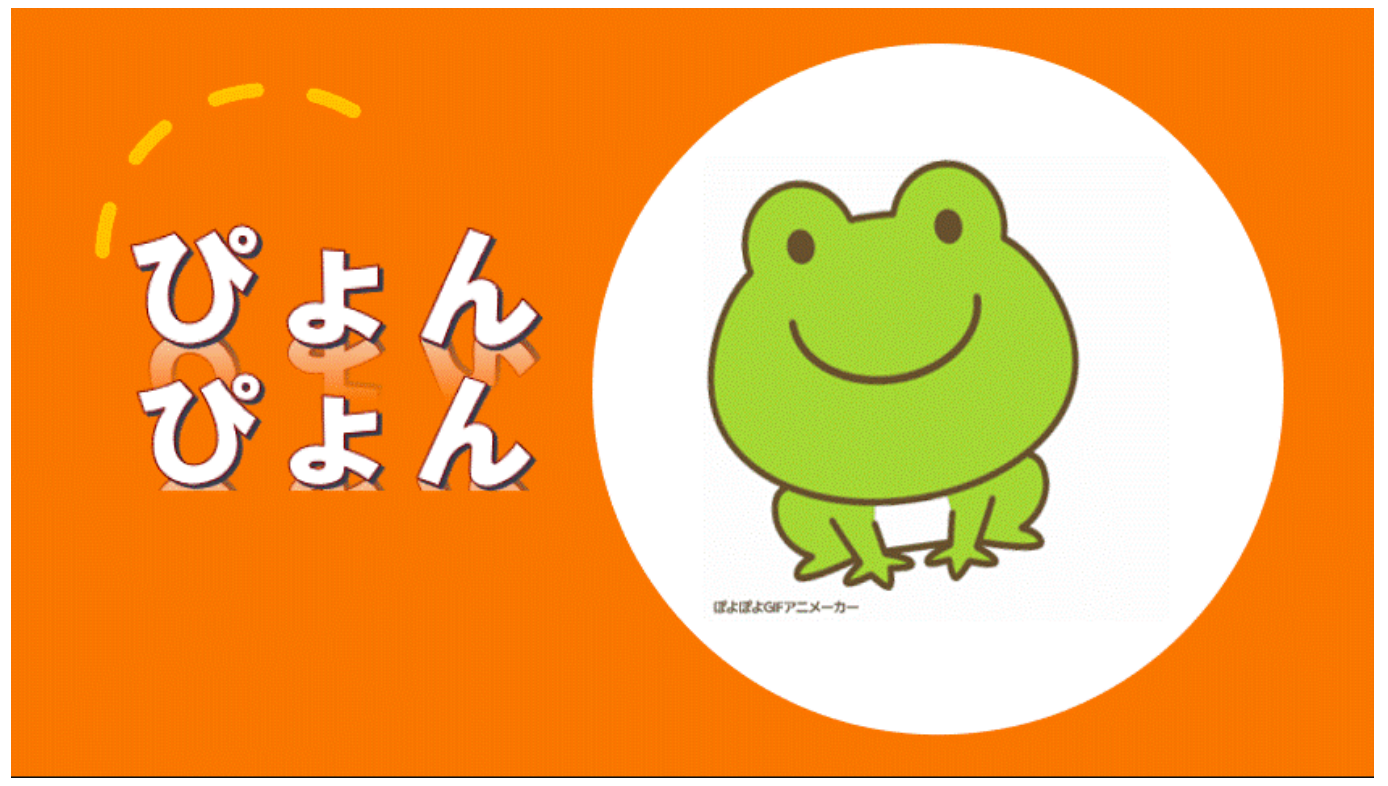

Para los aprendices del japonés, es esencial dominar las onomatopeyas, pues éstas aparecen con gran frecuencia en los animes y mangas que tanto gustan a 
los estudiantes de japonés. Asimismo, son palabras imprescindibles para poder hablar como un nativo.

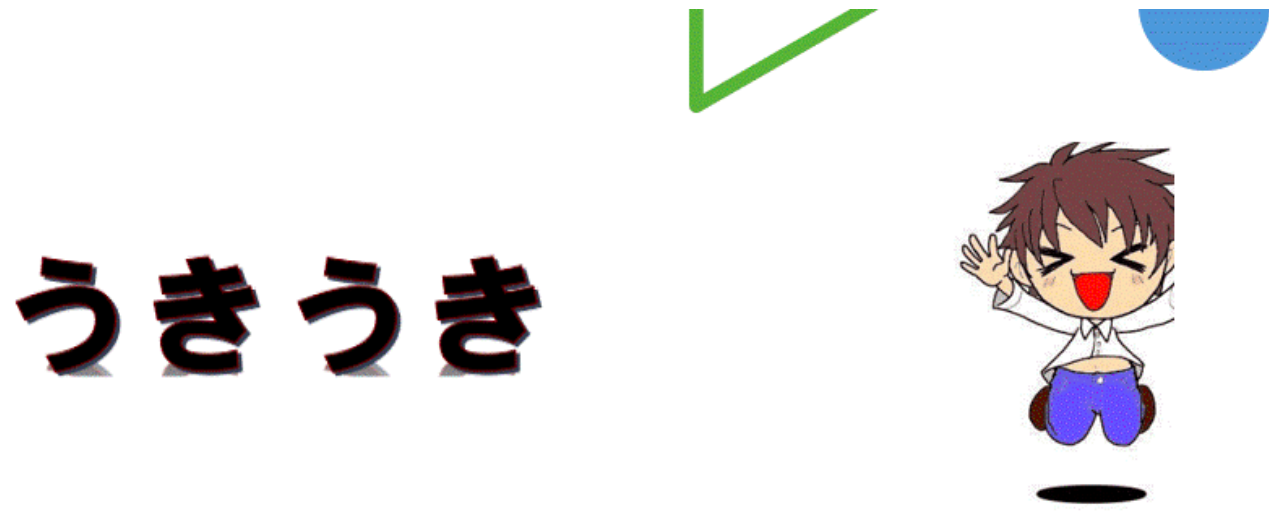

Imagen 5. Uki uki. Onomatopeya de cuando se está muy contento.

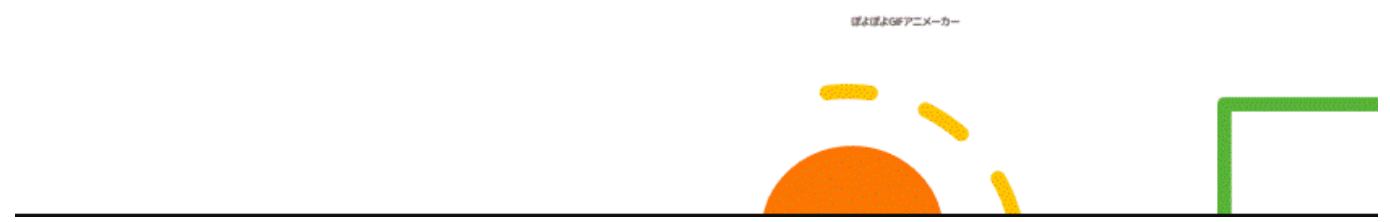

Gracias a mi participación en el taller de "Herramientas para la educación docente. De lo oral a lo digital", pude materializar la idea que tenía en mente acerca de las onomatopeyas en el japonés. Próximamente tomará forma y acción, además de en este artículo, al ser publicado en las redes sociales de la mediateca de la unAm.

\section{Páginas de interés}

- Japonés en el anime y manga

- Onomatopeinto

- Asesorías de japonés en la Mediateca de la ENALLT

- Padlet con las onomatopeyas de este artículo

\section{Referencias}

* Real Academia Española (RAe). (2014). Onomatopeya. En Diccionario de la lengua española (23.a ed., versión 23.4). https://dle.rae.es/onomatopeya

\section{Cómo CITAR ESTE ARTículo}

* Shimazaki Miho, Elisa Akemi. (2021, mayo-junio). Aprendizaje autodirigido en la educación superior: una perspectiva para la modalidad en línea. Revista Digital Universitaria (RDU), 22(3). http://doi.org/10.22201/cuaieed.16076079e.2021.22.3.13 